\title{
Q.
QNEEN'S
UNIVERSITY
BELFAST
}

\section{Visualising reactive oxygen species in live mammals and revealing of ROS-related system}

Guo, J., Xu, H., Wang, Z., Liu, S., Dai, Y., Lu, J., Zheng, S., Xu, D., Zhou, J., Ke, L., Cheng, X., Xu, M., Zhang, X., Guo, Y., Lin, Y., Ding, W., Gao, G., Wang, H., Chen, Q., ... Rao, P. (2019). Visualising reactive oxygen species in live mammals and revealing of ROS-related system. Free Radical Research. https://doi.org/10.1080/10715762.2019.1677902

Published in:

Free Radical Research

Document Version:

Peer reviewed version

Queen's University Belfast - Research Portal:

Link to publication record in Queen's University Belfast Research Portal

Publisher rights

(c) 2019 Informa UK Limited, trading as Taylor \& Francis Group. This work is made available online in accordance with the publisher's policies. Please refer to any applicable terms of use of the publisher.

\section{General rights}

Copyright for the publications made accessible via the Queen's University Belfast Research Portal is retained by the author(s) and / or other copyright owners and it is a condition of accessing these publications that users recognise and abide by the legal requirements associated with these rights.

Take down policy

The Research Portal is Queen's institutional repository that provides access to Queen's research output. Every effort has been made to ensure that content in the Research Portal does not infringe any person's rights, or applicable UK laws. If you discover content in the Research Portal that you believe breaches copyright or violates any law, please contact openaccess@qub.ac.uk. 


\section{Visualizing Reactive Oxygen Species in Live Mammals and Revealing of an ROS-Related System}

Jingke Guo ${ }^{\mathrm{a}, \mathrm{c}, 1}$, Hang $\mathrm{Xu}^{\mathrm{b}, 1}$, Shutao Liu ${ }^{\mathrm{c}, 1}$, Zicai Wang ${ }^{\mathrm{b}}$, You Dai ${ }^{\mathrm{a}}$, Jianzhi $\mathrm{Lu}^{\mathrm{b}}$, Shusen Zheng ${ }^{\mathrm{b}}$, Dazheng $\mathrm{Xu}^{\mathrm{b}}$, Jianwu Zhou ${ }^{\mathrm{a}}$, Lijing Ke ${ }^{\mathrm{a}}$, Xi Cheng ${ }^{\mathrm{d}}$, Mingming Xü, Xin Zhang ${ }^{\mathrm{b}}$, Yi Guo ${ }^{\mathrm{b}}$, Yingjie Lin ${ }^{\mathrm{b}}$, Wei Ding ${ }^{\mathrm{a}}$, Guanzhen Gao $^{\mathrm{a}}$, Huiqin Wanga ${ }^{\mathrm{a}}$, Qi Chen ${ }^{\mathrm{a}}$, Xiaowei Yu ${ }^{\mathrm{a}}$, Han Chen ${ }^{\mathrm{a}}$, Lina Qin ${ }^{\mathrm{f}}$, Xicui Sun $^{\mathrm{b}}$, Zhe Li ${ }^{\mathrm{b}}$, Shuyu Zheng ${ }^{\mathrm{b}}$, Jiaqi Wang ${ }^{\mathrm{b}}$, Yanglei Cheng ${ }^{\mathrm{b}}$, Shuai Qiu ${ }^{\mathrm{g}}$, Yuqiu $\mathrm{Hu}^{\mathrm{b}}$, Penghan Huang ${ }^{\mathrm{b}}$, Chuntong $\mathrm{Lin}^{\mathrm{c}}$, Qiming $\mathrm{Wu}^{\mathrm{h}}$, Yubo $\mathrm{Li}^{\mathrm{i}}$, Tianbao Chen ${ }^{\mathrm{j}}$, Chris Shaw ${ }^{\mathrm{j}}$, Sherry Ho ${ }^{\mathrm{b}}$, Qiang Wang ${ }^{\mathrm{k}}$, Huaiyu $\mathrm{Gu}^{\mathrm{b},{ }^{*}}$ and Pingfan $\mathrm{Rao}^{\mathrm{a}, \mathrm{c}, *}$

a Shanghai Institutes for Biological Sciences Chinese Academy of Sciences-Zhejiang Gongshang University Joint Centre for Food and Nutrition Research, Zhejiang Gongshang University, Hangzhou, Zhejiang 310035, China

b Department of Anatomy, Zhongshan School of Medicine, Sun Yat-Sen University, Guangzhou, Guangdong 510080, China

${ }^{\mathrm{c}}$ Institute of Biotechnology, Fuzhou University, Fuzhou, Fujian 350000, China

${ }^{\mathrm{d}}$ College of Medicine and Life Sciences, University of Toledo, Toledo, OH 43614, USA

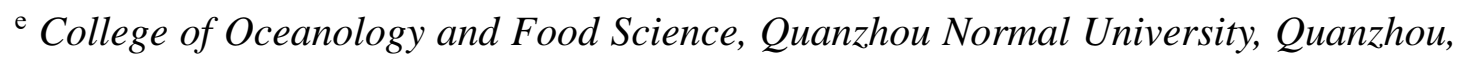
Fujian 362000, China

${ }^{\mathrm{f}}$ Department of Histology and Embryology, Zhongshan School of Medicine, Sun Yat-sen University, Guangzhou, Guangdong 510080, China

${ }^{\mathrm{g}}$ Department of Orthopedic and Microsurgery, Sun Yat-sen University First Affiliated 
Hospital, Guangzhou, Guangdong 510080, China

${ }^{\mathrm{h}}$ Graduate School of Agricultural Science, Tohoku University, Sendai, Miyagi 9800845, Japan

${ }^{i}$ College of Information Science and Electronic, Zhejiang University, Hangzhou, Zhejiang 310027, China

j Natural Drug Discovery Group, School of Pharmacy, Queen's University Belfast, Belfast, Northern Ireland BT97BL, United Kingdom

${ }^{\mathrm{k}}$ Institute of Food Science and Technology, Chinese Academy of Agricultural Sciences, Beijing 100000, China

${ }^{1}$ These authors contributed equally to this work.

* Correspondence to: Pingfan Rao, 149 Jiaogong Road, Hangzhou, Zhejiang 310035, China. Tel: +86 1375084 2288. pingfanrao@mail.zjgsu.edu.cn; Huaiyu Gu, 135 Xingangxi Road, Guangzhou, Guangdong 510080, China. Tel: +86 18927598966. gu_huaiyu@yahoo.com 


\title{
Visualizing Reactive Oxygen Species in Live Mammals and Revealing of an ROS-Related System
}

\begin{abstract}
Of all the aerobic respiration by-products, cytotoxic superoxide derived from mitochondrial-leaked electrons, is the only one known to be disposed of intracellularly. Is this fate the only destiny for mitochondrial-leaked electrons? When Cynomolgus monkeys were injected intravenously with reactive oxygen species (ROS) indicators, the connective tissues of dura mater, facial fascia, pericardium, linea alba, dorsa fascia and other body parts, emitted specific and intense fluorescent signals. Moreover, the fluorescent signals along the linea alba of SD rats, did not result from the local presence of ROS but from the interaction of ROS indicators with electrons flowing through this tissue. Furthermore, the electrons traveling along the linea alba of mice were revealed to originate from mitochondria. These data suggest that mitochondrial-leaked electrons may be transported extracellularly to a hitherto undescribed system of connective tissues, which is pervasively networked, electrically-conductive and metabolically-related.
\end{abstract}

Keywords: connective tissue; reactive oxygen species; mitochondria; Cynomolgus monkey; electron

\section{Introduction}

In mitochondrial aerobic metabolism [1], coupled to ATP production [2], electrons leak from the electron transport chain to oxygen to form superoxide anions, which subsequently trigger the generation of other more reactive ROS within and outside the mitochondria $[3,4]$. It is generally accepted that superoxide and the resultant ROS are 
neutralized by an antioxidant mechanism consisting of antioxidant enzymes and antioxidants within mitochondria and cells, through which cellular redox homeostasis is maintained [5].

While $\mathrm{H}_{2} \mathrm{O}$ and $\mathrm{CO}_{2}$, two mild metabolic by-products, are secreted from the mitochondria and cells to be eventually disposed of in lymphatic, circulatory and respiratory systems, cytotoxic superoxide anions are disposed of intracellularly by each individual cell without apparent involvement of any extracellular system [6]. Until now, this reasonable assumption has not been seriously questioned.

Oxygen radicals produced by epithelial cells post-infection, were found to induce paracrine activation of neighboring cells without involvement of gap junctions, cytokine secretions, ion fluxes, or nitric oxide synthesis. Oxygen radicals propagate extracellularly by an unknown mechanism, which may possibly be through electron transduction [7].

One hypothesis suggests that a tiny mitochondrial leak of electrons may be directly transported out of mitochondrial and cell membranes, constituting a novel signaling pathway [8]. This is on the basis of the unrivaled aptitude of electrons for signaling roles and the capability of intracellular semi-conducting electron transport and surface electron transfer within the cytoskeletal components $[9,10]$, to which mitochondria are connected [11].

The initial clue challenging the common belief was obtained in an attempt to visualize ROS distribution in live SD rats. When we injected rats intravenously with the ROS indicators, $2^{\prime}, 7^{\prime}$-dichlorofluorescein diacetate (DCFH-DA) and MitoSOX 
Red (MitoSOX) [12], intense fluorescent lines appeared on the frontal abdominal wall showing a pattern similar to that of classical acupuncture meridians. Neither unanimous, dispersed nor random, the specific fluorescent patterns indicated the unlikelihood of intracellular disposal of superoxide derived from mitochondrial leakage of electrons, and revealed the existence of a system related to ROS [13].

The existence of a ROS related system was confirmed by double blind clinical trials. Allergic rhinitis and simple obesity were treated by topical application at acupoints of TAT-Cu,Zn superoxide dismutase (TAT-SOD) [14], an intracellular superoxide quencher, which achieved efficacy rates $[15,16]$ comparable to acupuncture [17]. Acupuncture points are mostly located on fascia [18,19], and the substrate of TAT-SOD is superoxide [20]. The ROS related system may exist in fascia and the ROS involved is superoxide. Moreover, acupoint ST36 was stimulated by massage with and without TAT-SOD, acupuncture needle insertion, and injection of glutathione, while monitoring the electric potential difference (EPD) between two neighboring acupoints along the same meridian. The interventions of TAT-SOD massage and glutathione injection showed very similar EPD patterns to acupuncture, suggesting that acupuncture may function through a superoxide regulating mechanism, whose effect can be electrically-monitored [21].

In this study, the specific distribution of ROS in different mammals was investigated in order to elucidate the nature of the extracellular presence of ROS and associated biological implications, with the view to elucidating the logical fate of 
mitochondrial leaked electrons and perhaps a specific system devoted to this aspect of homeostasis.

\section{Materials and Methods}

\section{Animals}

Three male 7 year old Cynomolgus monkeys weighing 6.2-6.4 $\mathrm{kg}$ were purchased from Wincon TheraCells Biotechnologies Co., Ltd. (Nanning, Guangxi, China, SYXK(GUI)2014-0004). Fifty male specific pathogen-free (SPF) grade Sprague-Dawley (SD) rats weighing 180-220 g and Ten male SPF grade C57BL/6 mice weighing 22-24 g were purchased from Zhejiang Laboratory Animal Center, Zhejiang Academy of Medical Sciences (Hangzhou, Zhejiang, China, SYXK(ZHE)2014-0008). Twenty male SPF grade C57BL/6-TgH(mito-cpYFP) mice weighing 22-24 g were purchased from Cyagen (Guangzhou, Guangdong, China, SCXK(YUE)2013-0032). The animals were housed under barrier-sustained conditions at $25^{\circ} \mathrm{C}$ with a $12 \mathrm{~h}$ light/dark cycle and ad libitum access to water and food for $5 \mathrm{~d}$ prior to the operation.

All animals used in this present study were treated according to the Guide for the Care and Use of Laboratory Animals published by the National Institutes of Health. The protocol was approved by the institutional animal care and use committee of Wincon TheraCells Biotechnologies Co., Ltd. (approved protocol document \#: IACUC-05-A, protocol \#: WD-20170505, IACUC\#: W00124) and the animal experiment ethics committee of Zhejiang Academy of Medical Sciences (approved 
protocol ID: 2016R10031). All surgical procedures were performed under inhalation anesthesia using isoflurane, and every effort was made to reduce suffering. All animals were sacrificed with deep anesthesia and bleeding caused by cutting inferior vena cava.

\section{Visualising ROS distribution in Cynomolgus monkeys}

After anesthesia, $25 \mathrm{mg} / \mathrm{kg}$ DCFH-DA (Sigma-aldrich, St. Louis, Missouri, USA) and $2.5 \mathrm{mg} / \mathrm{kg}$ of MitoSOX (Thermo Fisher Scientific, Eugene, Oregon, USA) were dissolved in $5 \mathrm{ml} / \mathrm{kg}$ dimethyl sulfoxide (Sinopharm Chemical Reagent, Shanghai, China), then injected in small saphenous vein of Cynomolgus monkeys in 30 minutes. 30 minutes after injection, the skin was removed off the main anterior body to observe ROS distribution in forehead, face, chest, abdomen and perineum, and then the skull, thoracic cavity and abdominal cavity were open for the observation of relevant organs and tissues. The exposed parts were minutely incised. The fluorescent imaging was carried out with the exciting lights, which were generated by an UltraFire MCU WF-1200L lamp through band-pass filters of $488 \mathrm{~nm}$ for DCFH-DA and $510 \mathrm{~nm}$ for MitoSOX reagent, respectively. The fluorescence images were collected by a NIKON (model D-80) camera equipped with band-pass filter of $525 \mathrm{~nm}$ for DCFH-DA and $588 \mathrm{~nm}$ for MitoSOX reagent, respectively. The experiments were repeated 3 times.

Immunohistochemical and microscopic investigation of the fluorescent linea alba was conducted on a sample of $1 \times 1 \times 3 \mathrm{~mm}$ after incubation with DAPI for visualizing cell nucleus, $\mathrm{Cy} 3$ for collagen, then observed with a transmission electron microscope (TEM, FEI, Valley City, ND, USA) at a voltage of $80 \mathrm{KV}$. 


\section{Structural properties of the concentrated ROS distribution in linea alba of SD rats}

To identify elements responsible for the fluorescent light emission, linea alba of SD rats were studied. After anesthesia, $1 \mathrm{ml}$ of DCFH-DA solution (5 mg in $1 \mathrm{ml}$ DMSO) was injected into the tail vein. 30 minutes later, the frontal abdominal wall was incised, and further cross sectioned for the examination by a microscope (Leica, Buffalo Grove, Illinois, USA). The experiments were repeated 3 times.

\section{Electric properties of the concentrated ROS distribution in linea alba of SD rats}

$1 \mathrm{ml}$ of DCFH-DA solution (0.05 $\mathrm{mg}$ in $1 \mathrm{ml} \mathrm{DMSO})$ was smeared directly on the linea alba on the interior surface of an isolated frontal abdominal wall with and without the reconnecting linea alba by electric wire to the cutting points (xiphoid and symphysis pubis). A digital ammeter was used to measure the current between the xiphoid and symphysis pubis on the linea alba. The experiments were repeated 3 times.

\section{Measurement of electric current in linea alba of mice}

The mice were anesthetized. The abdominal walls of mice were incised. A digital ammeter was used to measure the current between the xiphoid and symphysis pubis on the linea alba. The symphysis pubis was marked with a positive terminal. The linea alba current was measured with three groups of mice:

cpYFP- group: 6 male C57BL/6 mice, weight $22.7 \pm 0.8 \mathrm{~g}$. Connect the body to an hour later, $300 \mu 1$ deionized water drops were dripped on liver. 
cpYFP+ group: 6 male C57BL/6-TgH(mito-cpYFP) mice, weight $21.2 \pm 1.3 \mathrm{~g}$. Connect the body to an hour later, $300 \mu \mathrm{l}$ deionized water drops were dripped on liver.

cpYFP+H2O2 group: 6 male C57BL/6-TgH(mito-cpYFP) mice, weight $21.3 \pm 1.5$ g. Connect the body to an hour later, $300 \mu 110 \mathrm{mM} \mathrm{H}_{2} \mathrm{O}_{2}$ drops were dripped on liver.

\section{Statistical analysis}

Because of the complex design of this pilot study, sample size was chosen on the basis of practical considerations. Therefore, this study was not designed to have sufficient power, and the results of statistical testing have to be interpreted as descriptive, explorative, and hypothesis generating rather than as confirmatory. Data are reported as means \pm SD. The statistical analysis was performed with SPSS 15.0 (SPSS, Chicago, USA) and R version 2.9.0 software.

\section{Results}

\section{Visualising ROS in live Cynomolgus monkeys}

The experiments were repeated 3 times, and the representative data are presented here. Injection of a mixture of DCFH-DA and MitoSOX resulted in patterns of emitted green or red fluorescent light in various body parts of Cynomolgus monkeys, as shown in Fig 1. Images of green and red fluorescence almost overlapped but with some subtle differences in some tissues. The intensity of the fluorescence can be considered as a measurement of ROS. In comparison with rats [13], the first images of 
ROS fluorescence in Cynomolgus monkeys were significantly more intense, likely due to the higher level of ROS production in the primates.

Various organs and tissues emitted ROS fluorescence with various intensities. Only fluorescent sites along the central line of the body are shown in Fig 1.

The intense fluorescence surrounding the eyes shows precisely where the oxidative stress in these organs is located. The anatomically discrete philtrum was revealed as a point with intense ROS activity, providing an interesting parallel with its role as a vital acupuncture point in traditional Chinese medicine (Fig 1A and 1B).

There was a sharp contrast between the dark heart and the intensely-fluorescent pericardium. The heart appeared almost oxidative stress-free, while the static pericardium was filled with ROS. Reliable and optimum heart performance requires this organ to be stress free, which is ensured by a potent oxidative stress buffering function, a previously unknown function for pericardium in addition to its role in pure physical and/or mechanical protection (Fig 1C and 1D).

As in the SD rat [13], the linea alba of Cynomolgus monkeys was also where ROS were concentrated. The linea alba of the SD rat passes through both surfaces of the frontal abdominal wall, and in Cynomolgus monkeys, this consists of three layers of muscles, hence 4 layers of fascia. In spite of such fundamental differences, both animals possess a ROS pathway in this structure, indicating its importance in mammals (Fig 1E and 1F).

Both small and large intestines were fluorescent, with the former being much more intense. It was verified that the fluorescence was emitted by the loose 
connective tissue layer of the intestinal wall (data not shown). Judging by the intensity of the fluorescence, the small intestine appeared to be more oxidatively-stressed than the large intestine.

In images of dura mater, the whole brain emitted fluorescence. The intensity in white matter was higher than that in grey matter with remarkably high intensity in the cord. This corresponds to the higher metabolism of white matter when compared with grey matter, and indicates the prominent presence/passage of ROS in the cord (Fig $1 \mathrm{G}$ and $1 \mathrm{H})$.

The dorsal fascia represent the physical site where low back pain frequently occurs. Remarkably intense green fluorescence suggests that this is the dominant location for ROS (Fig 1I and 1J). ROS have been associated with low back pain indirectly and the identification of high levels of ROS in the dorsal fascia provides evidence for this.

The area around the perineal central tendon was another important site for ROS. Adipose tissue emitted intense fluorescence (Fig 1K and 1L). Obviously, adipose tissue is not only used to store energy in the form of lipids while cushioning and insulating the body, but is also significantly implicated with ROS. These unexpected data present some pertinent evidence in support of the association of obesity with oxidative stress.

In summary, the detailed distribution of ROS in live Cynomolgus monkeys, revealed by small molecule ROS indicators (DCFH-DA and Mitosox), was from the top of the head down the face, and through the linea alba on the surface and different 
internal organs, to the perineal central tendon, and back through to the head through the dorsal fascia, completing a full circuit. ROS predominantly existed in dense and loose connective tissues like the fascia and in adipose tissues. The systemic presence and location of ROS has been confirmed in primates.

\section{Determination of histological components responsible for fluorescent light emission}

Linea alba of both SD rats and Cynomolgus monkeys, emitted intense ROS fluorescence. Microscopic and histological studies, were used to identify the exact anatomical components responsible for this fluorescence.

When SD rats were injected with DCFA-DA, green fluorescent lines emerged on the interior frontal abdominal wall, as shown in Fig 2B. The fluorescence was emitted by the linea alba and the linea semilunaris (Fig 2F). As is shown in the cross sectional images in Fig $2 \mathrm{C}$ and $2 \mathrm{E}$, in addition to the linea alba, the anterior and posterior layers of the rectus sheath and extraperitoneal fascia all emitted fluorescence (Fig 2D and 2E). Connective tissues could thus be identified as the specific tissue in which the fluorescence was present.

The fluorescent linea alba of Cynomolgus monkeys was also examined. Fig 3A shows the distribution of ROS, Fig 3B and Fig 3C for the immunohistochemical staining of nuclei and collagen, respectively. Both images indicated that cells and collagen were responsible for the fluorescence. Connective tissues contain fibroblasts and immune cells, both of which may contribute to the fluorescence. The presence of 
collagen fibers was further confirmed by the typical collagen fiber pattern in the TEM image shown in Fig 3E.

\section{ROS fluorescence resulted from electrons transmitted along the linea alba of SD rats.}

To elucidate the mechanism of how connective tissues generate ROS fluorescence, ROS visualization was performed on live SD rats under various conditions.

Incision of the frontal abdominal wall in SD rats and smearing DCFH-DA on the interior linea alba, did not produce any fluorescence (Fig 4C). This indicated that the linea alba either did not contain ROS or its content was not sufficient to emit fluorescence. However, after the cleaved frontal abdominal wall was re-connected to the rat body at the original cutting points of the linea alba by crocodile clips and wire (Fig 4A), smearing DCFH-DA on the interior linea alba generated intense fluorescence (Fig 4D). All other combinations of connecting points between the cleaved frontal abdominal wall and the rat body failed to generate fluorescence on the linea alba upon the indicator smearing (Fig 4E-H). The conduction only occurred along the linea alba. The linea alba did not produce fluorescence when the connecting points were switched. The linea alba thus has semiconducting properties and though not shown here, the same data were obtained using mice.

\section{Electric current in the linea alba of mice originated from mitochondria}

Exogenous $\mathrm{H}_{2} \mathrm{O}_{2}$ can lead to a increased production of ROS by a self-amplifying mechanism, including mitochondrial superoxide, GSH depletion, cellular $\mathrm{Ca}^{2+}$ signal transfer and intracellular ROS dynamic increase. The 
mitochondria has been reportedly identified to be the main origin or contributor of ROS bursts [22,23]. It is therefore possible to consider the ROS burst resulted from exogenous $\mathrm{H}_{2} \mathrm{O}_{2}$ stimulation as an indicator of mitochondrial ROS burst. To verify a mitochondrial origin for the electric current that generates ROS fluorescence in the linea alba, C57BL6-TgH(mito-cpYFP) mice were constructed, in which cpYFP, a ROS reporting fluorescent protein, was expressed in mitochondria of various tissues [24]. As an electron donor to ROS, cpYFP expression could be expected to influence electrical output from mitochondria, and hence can be used to identify any connection between mitochondria and electric current transmitted in the linea alba.

The frontal abdominal walls were excised from wild type mice (C57BL/6, group mito-cpYFP-) and transgenic mice (C57BL6-TgH, group mito-cpYFP+), respectively. A DC ampere meter was connected (Fig 5A) to measure current in the linea alba. While around $0.7 \mu \mathrm{A}$ was detected in wild type mice, very low current was detected in their transgenic counterparts. However, stressing transgenic mice by dripping $300 \mu \mathrm{l} 10 \mathrm{mM} \mathrm{H}_{2} \mathrm{O}_{2}$ solution onto their livers, instantly increased current in the linea alba of transgenic mice (C57BL6-TgH, group mito-cpYFP $+\mathrm{H} 2 \mathrm{O} 2$ ) from 0 to around $0.3 \mu \mathrm{A}$. The linea alba current responded sensitively to changes in mitochondrial conditions, such as in the systemic expression of cpYFP or individual organ chemical stress, revealing an electrical connection between mitochondria and the linea alba and/or other connective tissues. 


\section{Discussion}

DCFH-DA and other fluorescent probes react to various ROS with low specificity. The fluorescence alone provides no information for ROS type identification. Results of the previous clinical trials of topical application of TAT-SOD to acupuncture points suggest the involvement of fascia as well as superoxide as the ROS type $[15,16]$. Whilst connective tissue was confirmed to be the source of fluorescence, superoxide should be the ROS which effectively oxidizes the probes. What is unusual is that the superoxide anions which produced the fluorescence in the connective tissue are neither generated locally nor exist in a constant high concentration in this tissue (Figs 1 and 2).

The linea alba contains both cells and capillaries in far lower densities than those found in adjacent muscle tissues and its metabolic intensity and hence ROS production, should thus be lower. Since muscle tissue of higher metabolic intensity and ROS content did not emit detectable fluorescence, ROS generated by connective tissue were much less likely to contribute to this. On the other hand, the fluorescence emitted by the linea alba was so intense, that it was comparable to the levels emitted by the edge of mechanically-damaged tissue. High levels of ROS or superoxide anions can result in cell and tissue destruction but as the histological structure of the linea alba remained unchanged while emitting intense ROS fluorescence, this would suggest a transient nature of ROS or superoxide anions in this tissue (Figs 2 and 3). 
While connective tissues were readily identified as the source of ROS fluorescence, the fluorescence generating mechanism must be different from what is known for the fluorescent probes (Fig 3).

As is apparent from the above results (Fig 4), the linea alba transmitted electrical currents unidirectionally, and the conducted electrons behaved like ROS to oxidize DCFH-DA to generate fluorescence. Connective tissues contain both structural proteins including collagen, and cells such as fibroblasts and immune cells (Fig 3). Fibroblasts, collagen fibers and the cytoskeleton are reportedly to play cell-cell signaling roles for ROS [7,25], capable of electrical conduction [26,27], and hence possibly constitute the conducting components of connective tissue. It is of interest that electron transmissions in these mimic ROS to deprive DCFH-DA of electrons. Intracellular semiconduction electron transport and surface electron transfer are possible in macromolecules [8]. It is possible that collagen fibers and cellular components possess ROS capability of electron hopping or surface electron transfer possesses an ROS-equivalent energy level that facilitates the transfer of electrons from DCFH. In fact, when a $1.5 \mathrm{~V}$ battery current was applied to the linea alba replacing the electric output of the rats, no conduction occurred and consequently no fluorescence could be generated, confirming the specificity of the transmitted electron.

The identification of the source of electrical current transmitted in the linea alba and its biological significance are important. Bioelectricity can result from various sources such as cellular membrane potential and even ion concentration 
differences. In redox electron transfer, electron transfer is not driven by the energy potential difference but by the resonant energy level $[28,29]$. The ROS or superoxide-like behavior of the connective tissue suggests that connective tissue is likely a channel for the transferring of electrons of ROS or superoxide energy levels [30,31]. Mitochondrially-leaked electrons are the perfect candidates for this transfer due to their quantity in highly metabolizing tissues [32].

Among body structures such as blood vessels, lymphatic vessels and nerve fibers, collagen fibers and other structural proteins in the connective tissue are physically the most likely mediators in the electrical linkage. As structural support for tissues and organs, vessels, ducts and tubes, connective tissues constitute such a pervasive system capable of making many connections within the body [33]. The conductivity of collagen fibers has long been recognized. It has been demonstrated in this study that electron transmission by ROS or superoxide energy levels, constitute a biomechanical system whose bioelectricity is specifically related to ROS. Mitochondria sit on the cytoskeleton, and can transmit electrons through connections to integrin and collagen fibers incorporated into the fascia, ligaments and so on. There is thus a ROS electrical transmission pathway from mitochondria to connective tissues, which is the reason why the expression of cpYFP and increased metabolic rate, could change the electrical current in the linea alba (Fig 5).

In mitochondria, electron flow occurs at various respiratory complexes due to electron leakage. Although there must be other sources of electron flow, mitochondrial-leaked electrons possess a superoxide redox energy level capable of 
transmission through the ROS electrical pathway in connective tissue from mitochondria to the linea alba.

It is unknown how mitochondrial-leaked electrons are physically collected and transported. Yet, it is reasonable to predict the presence of such a pathway from leakage sites of complexes out of the mitochondria or cytoskeleton and onwards. cpYFP must have been expressed on this ROS electron transmission pathway. As in connective tissues, electron transmission must have mimicked the properties of superoxide which oxidized cpYFP, by depriving an electron to neutralize its own unpaired electron, thus diminishing the total electron transmission, consequently resulting in reduced electrical current in the linea alba. On the other hand, the sharp and instant increase in the linea alba current occurred when the transgenic mice were subject to the toxic challenge to liver with hydrogen peroxide. This could be attributed to an increased electron leakage in mitochondria [8] with the elevated metabolic rate [34,35]. All results indicate that the electrons transmitted in the linea alba and other connective tissues are those leaked from the mitochondria.

It is not known if electron leakage is an imperfection or a process with a specific biological purpose. For the pure purpose of waste disposal, it makes perfect sense that mitochondrial-leaked electrons are transmitted out of the mitochondria and cells to a system of dispersal/disposal. That is how the less toxic $\mathrm{H}_{2} \mathrm{O}$ and $\mathrm{CO}_{2}$ by-products are disposed of [8]. Moreover, the amount of ROS generated in normal metabolism is not trivial. If $2 \%$ [36] inhaled oxygen [37] is turned into superoxide [11], the total electric current resulting from mitochondrial leaked electrons can reach 
$1.08 \mathrm{~A}$ in an adult! It is reasonable therefore to speculate that the greatest proportion of leaked electrons are transmitted out of mitochondria and cells for disposal while a small fraction goes through the intra-mitochondrial antioxidant cascade, which should be an auxiliary mechanism but not the only or indeed major one.

If the quantity of mitochondrial leaked electrons is proportional to ATP generation, transporting these out of cells rather than utilizing intra-mitochondrial neutralization would make the most reliable and instant aberrant electrical signal disposal system for cellular metabolism. It may be exactly this very specific biological function that mitochondrial leaked electrons have been entrusted with.

In fact, from mitochondria to cell membrane and then to the collagen fiber of connective tissue, there is a pathway by linking to cytoskeleton, actin and integrin on the membrane and then on to collagen with some connecting proteins [38,39]. All the connecting components are macromolecules and their assembled structure capable of electron conduction $[8,40]$. It should be possible to verify it as a pathway to transmit the leaked electrons out of mitochondria to the cell membrane, and on to connective tissue system.

ROS distribution evidence in this study were collected from mice, SD rats and Cynomolgus monkeys according to experimental restrictions and convenience. It may be imprudent to make a general reasoning based on such evidence, but what is consistent across the species is the new insight generated concerning connective tissue involvement with ROS and its conductivity of superoxide related electrons, namely mitochondrial-leaked electrons. The high specificity makes the connective tissue a 
highly reliable electrical circuit exclusively for mitochondrial leaked electrons or metabolic signaling.

From molecular interaction to electron transmission, from intracellular neutralization to systemic disposal, from biomechanical to bioelectric, imaginative research efforts are required in order to complete this leap of faith with regards to this new functional organ system. It will also widen the understanding of diseases from the focus on visceral organs to their connections, and may provide simple and powerful tools to unlock the pathogenesis of some intractable and incurable diseases.

\section{Conclusions}

The connective tissue network is ROS channels, which is bioelectric related to mitochondria, may possibly be an electric circuit regulating metabolism. Our findings suggest that disposal by a system involving a network of connective tissues can be a more logic fate for mitochondrial leak electrons, and a metabolism-related electric circuit of fascia sound more reasonable to handle metabolic signals than molecular diffusion and interactions. Most excitingly, decoding the patterned ROS distribution may provide tangible clues to bioelectromagnetics, which can provide insights of biology beyond biomolecules.

\section{Acknowledgments}

We thank Fundamental Life Sciences Company Limited, Hongkong for technical supports and Huanhuan Zhang of the Zhejiang Academy of Medical Sciences for expert help in data acquisition. 


\section{Funding}

This work was supported by the National Key R\&D Program of China under Grant No. 2016YFD0400202; National Natural Science Foundation of China under Grant Nos. 31500685 and 31271859; Zhejiang Provincial Natural Science Foundation of China under Grant No. LY16C050002 and Start-Up Fund of Research in Quanzhou Normal University, China under Grant No. G16062.

\section{Disclosure statement}

The authors report no conflict of interest.

\section{Data availability statement}

The data that support the findings of this study are openly available in Mendeley Data at http://dx.doi.org/10.17632/jm3w3gv2px.3.

\section{References}

1. Saran M. To what end does nature produce superoxide? NADPH oxidase as an autocrine modifier of membrane phospholipids generating paracrine lipid messengers. Free Radic Res. 2003;37(10):1045-1059.

2. Raha S, Robinson BH. Mitochondria, oxygen free radicals, disease and ageing. Trends Biochem Sci. 2000;25(10):502-508.

3. Suh YA, Arnold RS, Lassegue B, et al. Cell transformation by the superoxide-generating oxidase Mox1. Nature. 1999;401(6748):79-82.

4. Chance B, Sies H, Boveris A. Hydroperoxide metabolism in mammalian organs. Physiol Rev. 1979;59(3):527-605. 
5. McCord JM, Fridovich I. Superoxide dismutase: the first twenty years 1968-1988. Free Radic Biol Med. 1988;5(5-6):363-369.

6. Geers C, Gros G. Carbon dioxide transport and carbonic anhydrase in blood and muscle. Physiol Rev. 2000;80(2):681-715.

7. Dolowschiak T, Chassin C, Ben Mkaddem S, et al. Potentiation of epithelial innate host responses by intercellular communication. PLoS Pathog. 2010;6(2):e1001194.

8. Oschman JL. Charge transfer in the living matrix. J Bodyw Mov Ther. 2009;13(3):215-218.

9. Cooper SK, Pandhare J, Donald SP, Phang JM. A novel function for hydroxyproline oxidase in apoptosis through generation of reactive oxygen species. J Biol Chem. 2008;283(16):10485-10492.

10. Phang JM, Liu W, Zabirnyk O. Proline metabolism and microenvironmental stress. Annu Rev Nutr. 2010;30:441-463.

11. Isenberg I. Free radicals in tissue. Physiol Rev. 1964;44:487-517.

12. Kalyanaraman B, Darley-Usmar V, Davies KJ, et al. Measuring reactive oxygen and nitrogen species with fluorescent probes, challenges and limitations. Free Radic Biol Med. 2012;52(1):1-6.

13. Guo J, Liu S, Cheng X, et al. Revealing acupuncture meridian-like system by reactive oxygen species visualization. Biosci Hypotheses. 2009;2(6):443-445.

14. Ye N, Liu S, Lin Y, Rao P. Protective effects of intraperitoneal injection of TAT-SOD against focal cerebral ischemia/reperfusion injury in rats. Life Sci. 2011;89(23-24):868-874.

15. Guo JK, Xu MM, Zheng MF, et al. Topical Application of TAT-Superoxide Dismutase in Acupoints LI 20 on Allergic Rhinitis. Evid Based Complement 
Alternat Med. 2016;2016:383027.

16. Guo J, Chen Y, Yuan B, Liu S, Rao P. Effects of intracellular superoxide removal at acupoints with TAT-SOD on obesity. Free Radic Biol Med. 2011;51(12):2185-2189.

17. Moore K, Roberts LJ. Effects of intracellular superoxide removal at acupoints with TAT-SOD on obesity. Free Radic Biol Med. 2011;51(12):2163.

18. Bai Y, Wang J, Wu JP, et al. Review of evidence suggesting that the fascia network could be the anatomical basis for acupoints and meridians in the human body. Evid Based Complement Alternat Med. 2011;2011:260510.

19. Yang C, Du YK, Wu JB, et al. Fascia and Primo Vascular System. Evid Based Complement Alternat Med. 2015;2015:303769.

20. Langevin HM, Yandow JA. Relationship of acupuncture points and meridians to connective tissue planes. Anat Rec. 2002;269(6):257-265.

21. Xu MM, Guo JK, Xu JS, et al. Monitoring the Effects of Acupoint Antioxidant Intervention by Measuring Electrical Potential Difference along the Meridian. Evid Based Complement Alternat Med. 2015;2015:286989.

22. Pan H, Wang BH, Li ZB, et al. Mitochondrial superoxide anions induced by exogenous oxidative stress determine tumor cell fate: an individual cell-based study. J Zhejiang Univ Sci B. 2019;20(4):310-321.

23. Park WH. Exogenous $\mathrm{H} 2 \mathrm{O} 2$ induces growth inhibition and cell death of human pulmonary artery smooth muscle cells via glutathione depletion. Mol Med Rep. 2016;14(1):936-942.

24. Fang H, Chen M, Ding Y, et al. Imaging superoxide flash and metabolism-coupled mitochondrial permeability transition in living animals. Cell Res. 2011;21(9):1295-1304. 
25. Waghray M, Cui Z, Horowitz JC, et al. Hydrogen peroxide is a diffusible paracrine signal for the induction of epithelial cell death by activated myofibroblasts. FASEB J. 2005;19(7):854-856.

26. Luin E, Giniatullin R, Sciancalepore M. Effects of $\mathrm{H}_{2} \mathrm{O}_{2}$ on electrical membrane properties of skeletal myotubes. Free Radic Biol Med. 2011;50(2):337-344.

27. Espinosa A, Leiva A, Peña M, et al. Myotube depolarization generates reactive oxygen species through NAD(P)H oxidase; ROS-elicited $\mathrm{Ca}^{2+}$ stimulates ERK, CREB, early genes. J Cell Physiol, 2006;209(2):379-388.

28. Kranich A, Ly HK, Hildebrandt P, Murgida DH. Direct observation of the gating step in protein electron transfer: electric-field-controlled protein dynamics. J Am Chem Soc. 2008;130(30):9844-9848.

29. Sakamoto K, Kamiya M, Imai M, et al. NMR basis for interprotein electron transfer gating between cytochrome $\mathrm{c}$ and cytochrome c oxidase. Proc. Natl. Acad. Sci. U S A. 2011;108(30):12271-12276.

30. Menon BR, Fisher K, Rigby SE, Scrutton NS, Leys D. A conformational sampling model for radical catalysis in pyridoxal phosphate- and cobalamin-dependent enzymes. J Biol Chem. 2014;289(49):34161-34174.

31. Leys D, Scrutton NS. Electrical circuitry in biology: emerging principles from protein structure. Curr Opin Struct Biol. 2004;14(6):642-647.

32. Cooper SK, Pandhare J, Donald SP, Phang JM. A novel function for hydroxyproline oxidase in apoptosis through generation of reactive oxygen species. J Biol Chem. 2008;283(16):10485-10492.

33. Benias PC, Wells RG, Sackey-Aboagye B, et al. Structure and Distribution of an Unrecognized Interstitium in Human Tissues. Sci Rep. 2018;8(1):4947.

34. Mekenyan O, Dimitrov S, Pavlov T, et al. Simulation of chemical metabolism for 
fate and hazard assessment. V. Mammalian hazard assessment. SAR QSAR Environ Res. 2012;23(5-6):553-606.

35. Parke DV, Sapota A. Chemical toxicity and reactive oxygen species. Int J Occup Med Environ Health. 1996;9(4):331-340.

36. Turrens JF. Mitochondrial formation of reactive oxygen species. J Physiol. 2003;552(Pt 2):335-344.

37. Hughson RL. Oxygen uptake kinetics: historical perspective and future directions. Appl Physiol Nutr Metab. 2009;34(5):840-850.

38. Ni HM, Williams JA, Ding WX. Mitochondrial dynamics and mitochondrial quality control. Redox Biol. 2015;4:6-13.

39. Westermann B. The mitochondria-plasma membrane contact site. Curr Opin Cell Biol. 2015;35:1-6.

40. Liu X, Liu H, Qu X, et al. Electrical signals triggered controllable formation of calcium-alginate film for wound treatment. J Mater Sci Mater Med. 2017;28(10):146. 


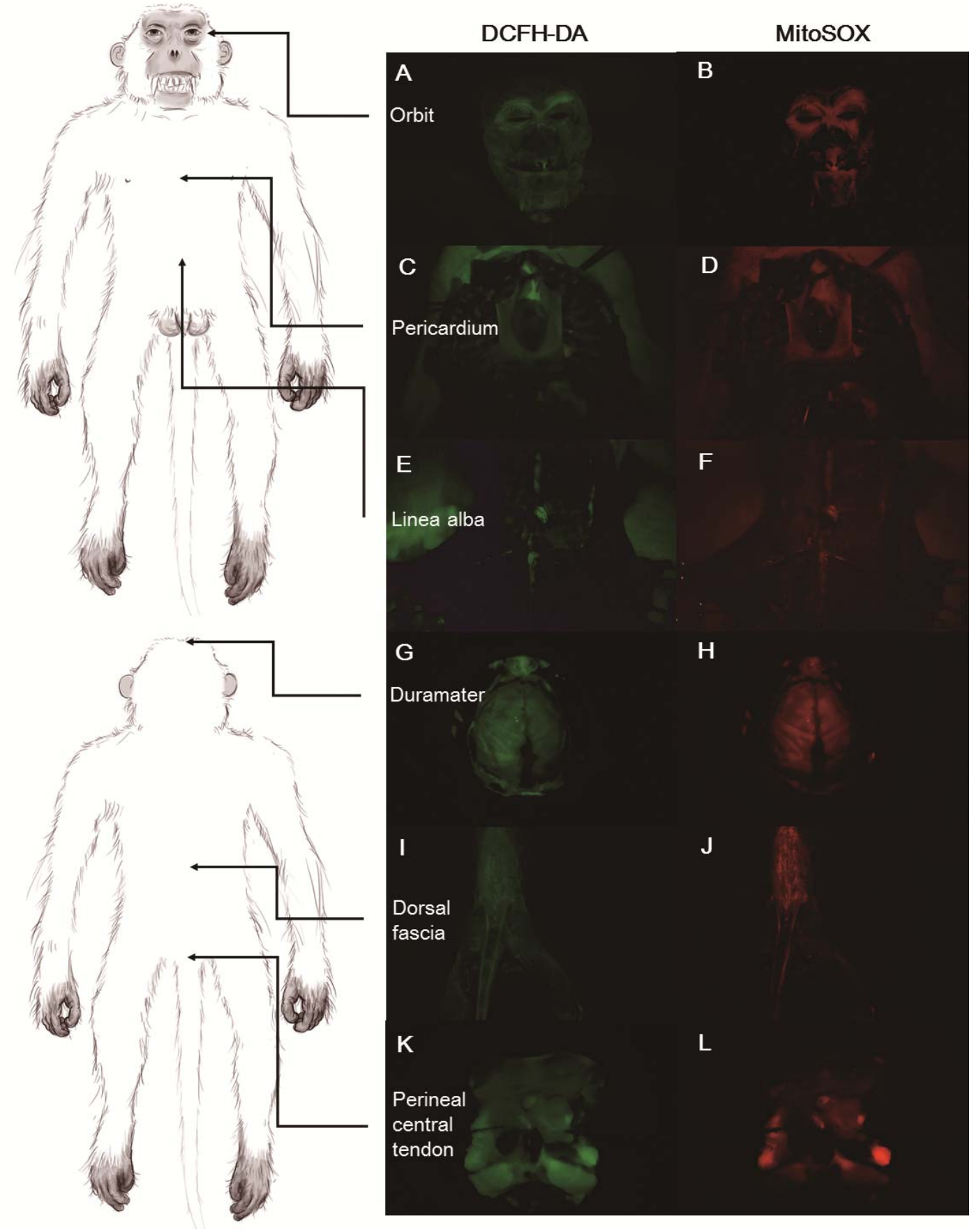

4 Figure 1. The fluorescent dyes, DCFH-DA and MitoSOX, were injected into 5 Cynomolgus monkeys through the venae saphena parva. Flourescence was observed 6 in regions of facial fascia (A and B), pericardium (C and D), linea alba (E and F), dura 7 mater $(\mathrm{G}$ and $\mathrm{H})$, back aponeurosis ( $\mathrm{I}$ and $\mathrm{I})$, perineal central tendon ( $\mathrm{K}$ and $\mathrm{L}$ ) and 8 other body parts with dense connective tissues. The exciting light was generated by an 9 UltraFire MCU WF-1200L lamp through a band-pass filter of $488 \mathrm{~nm}$ for DCFH-DA 
and $510 \mathrm{~nm}$ for MitoSOX reagent, respectively, and the image was collected by a NIKON (model D-80) camera equipped with a band-pass filter of $525 \mathrm{~nm}$ for DCFH-DA and $588 \mathrm{~nm}$ for MitoSOX reagent, respectively.

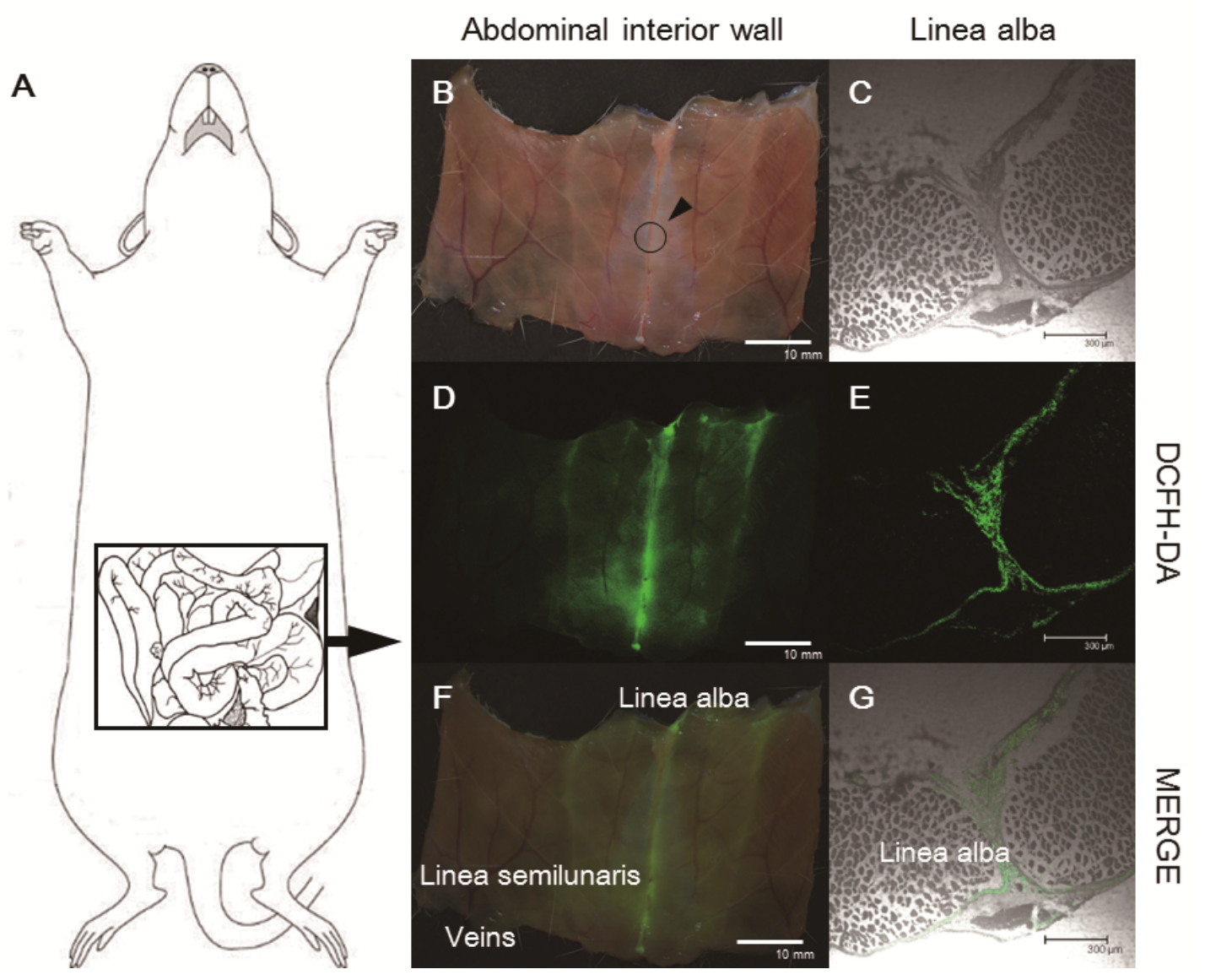

Figure 2. Images of the frontal abdominal interior wall of a male SD rat whose abdominal wall was incised (A). 30 minutes after the injection of $1 \mathrm{ml}$ of DCFH-DA solution (5 $\mathrm{mg}$ in $1 \mathrm{ml}$ dimethyl sulfoxide) through the tail vein (B, D and $\mathrm{F}$ ). Histological cross-sectional biopsy samples (C, E and G) from the area of the linea alba of the frontal abdominal wall. ROS were detected in living cells by the DCFH-DA fluorescent probe at excitation/emission wavelengths of 488/525 nm (green). 


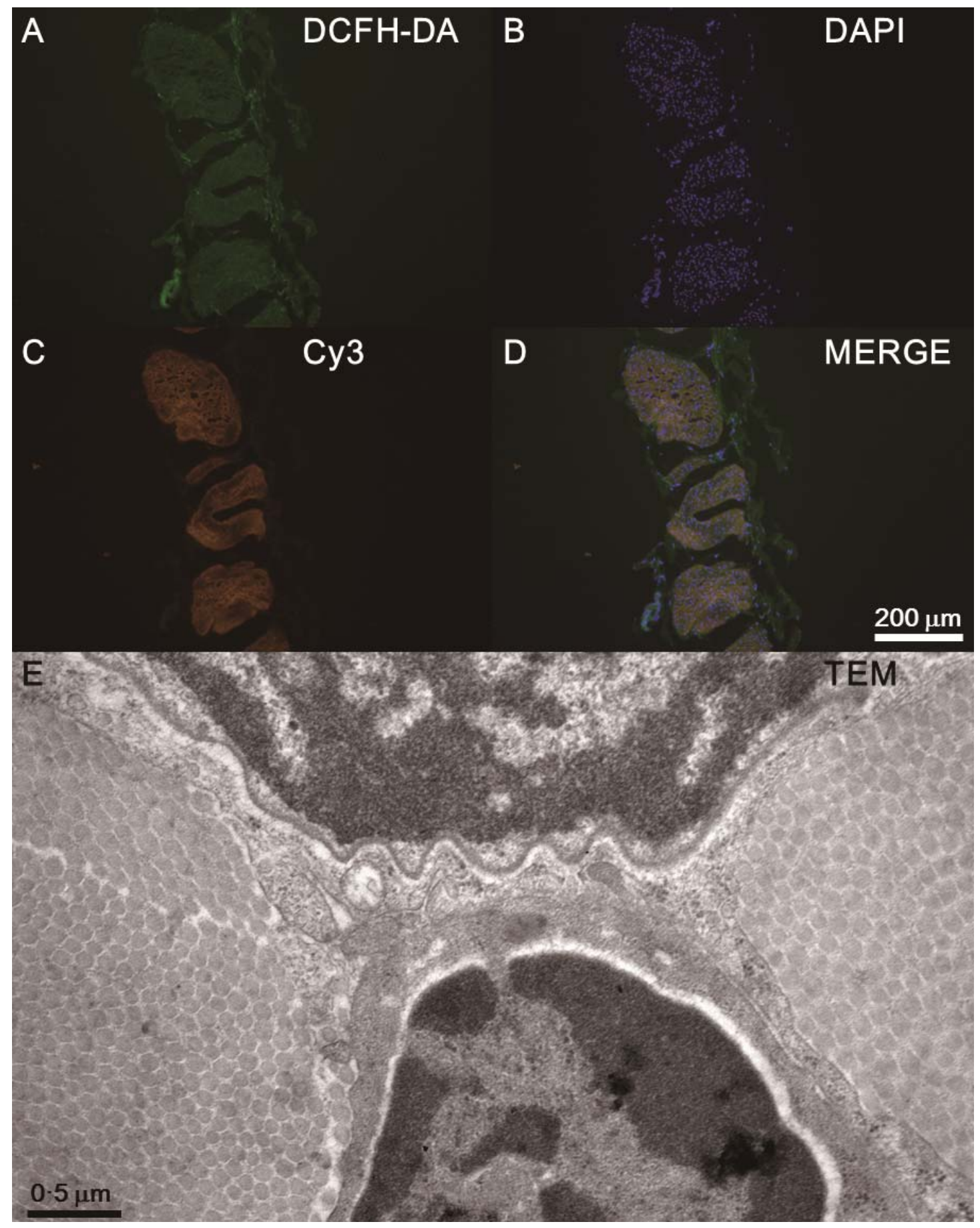

22 Figure 3. Immunohistochemical staining of linea alba in the Cynomolgus monkey.

23 Visualizing the distribution of ROS (A), cell nuclei (B), collagen (C). Transmission 24 electron microscopic (TEM) image of the fluorescent tissue showing the distribution 25 of collagen (E). The results confirmed that the fluorescence from the connective tissues coincided with the collagen fibers. 
A

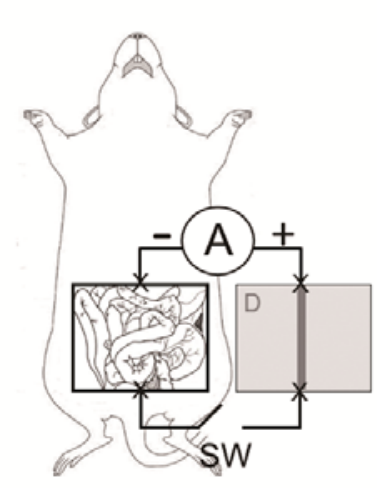

B 2.5

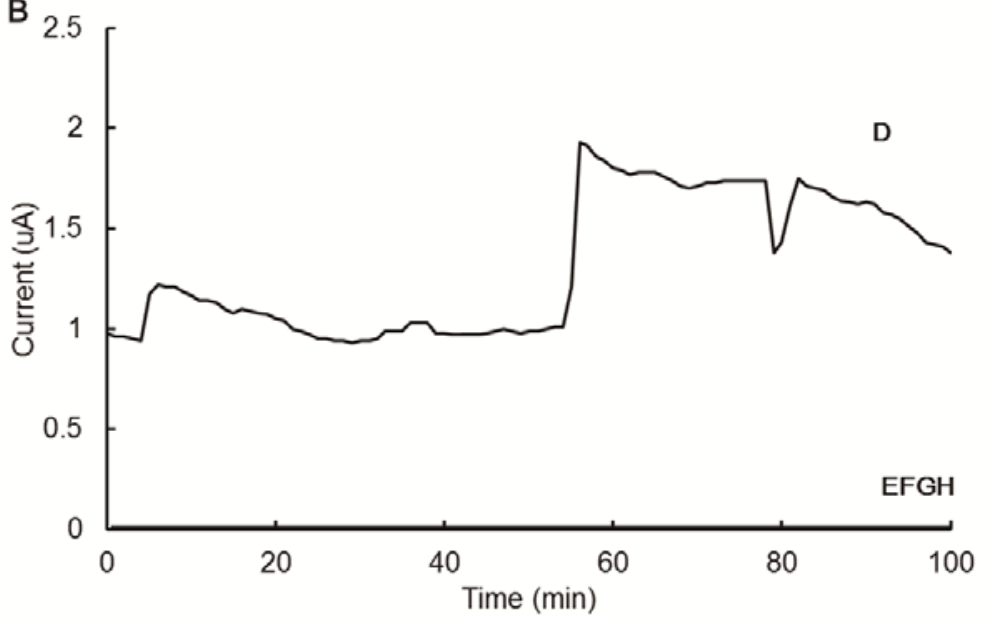

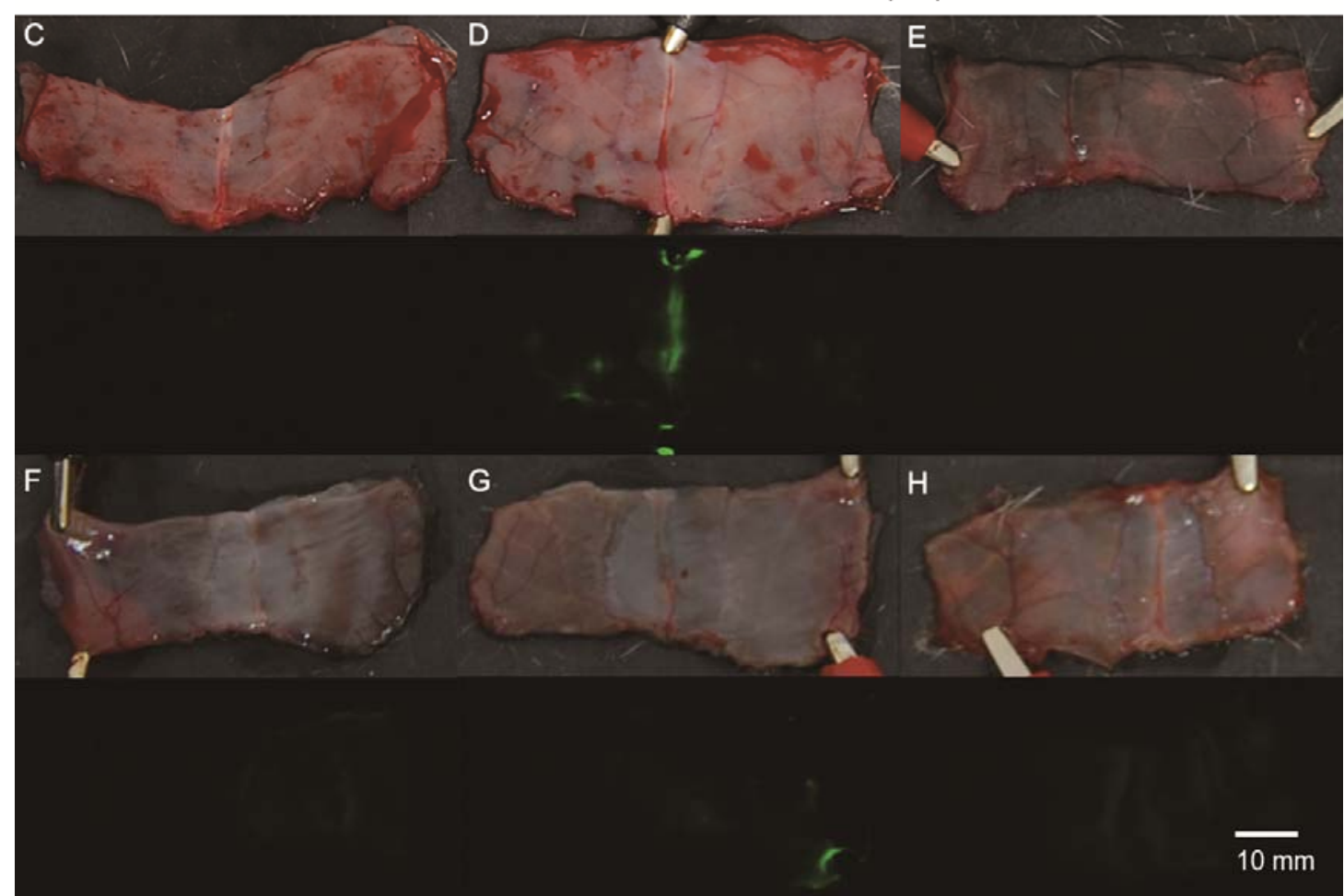

Figure 4. Images of the frontal abdominal interior wall of male SD rats whose abdominal walls were excised. SD rats linea alba (D) and other sites (E-H) of in-vitro abdominal wall was linked to xiphoid and symphysis pubis of body with a copper conductor (A). The other SD rat linea alba of in-vitro abdominal wall was unlinked (C). Strong fluorescent signals of ROS (D) and current (B) of the rat linea alba linked with the copper conductor could be detected, implying that the emergence of the fluorescence depends on electron transmission. ROS were detected in living cells by the DCFH-DA fluorescent probe at excitation/emission wavelengths of 488/525 nm (green). 

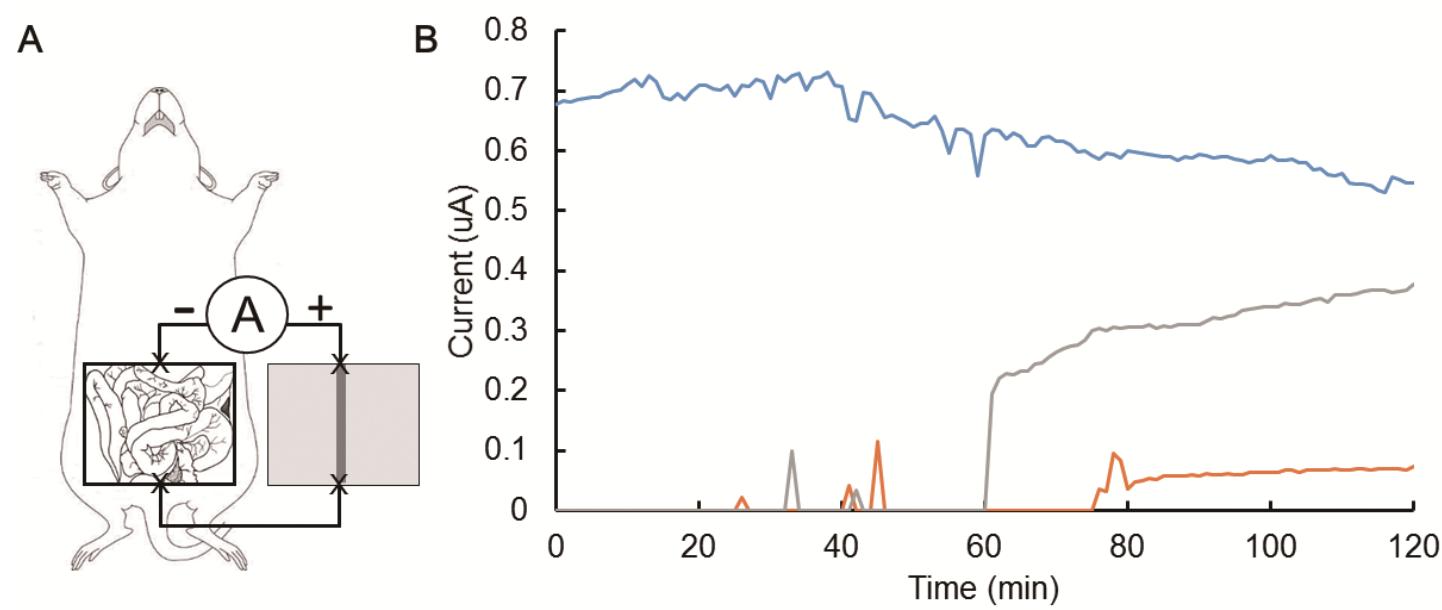

Figure 5. Monitoring the current between xiphoid and symphysis pubis of C57BL/6 39 mice (group cpYFP-) and C57BL6-TgH(mito-cpYFP) mice (group cpYFP+ and 40 group cpYFP $+\mathrm{H} 2 \mathrm{O} 2$ ). An hour later, $300 \mu$ deionized water (group cpYFP- and 41 group cpYFP+) or $10 \mathrm{mM} \mathrm{H}_{2} \mathrm{O}_{2}$ (group cpYFP+H2O2) drops were dripped on liver. 